ANNALES

POLONICI MATHEMATICI

$95.2(2009)$

\title{
Some envelopes of holomorphy
}

\author{
by Edgar Lee Stout (Seattle, WA)
}

\begin{abstract}
We construct some envelopes of holomorphy that are not equivalent to domains in $\mathbb{C}^{n}$.
\end{abstract}

1. Introduction. In [14] we exhibited domains in $\mathbb{C}^{n}, n \geq 2$, whose envelopes of holomorphy are not smoothly equivalent to domains in $\mathbb{C}^{n}\left(^{1}\right)$. The main purpose of the present note is to present an example of a domain, which lies in $\mathbb{C}^{7}$, whose envelope of holomorphy is real-analytically equivalent to a domain in $\mathbb{C}^{7}$ but is not biholomorphic to such a domain. The construction we use yields some other examples in the same spirit. The principal ingredients of the example are the known results that the seven-sphere $\mathbb{S}^{7}$ does not admit a totally real embedding in $\mathbb{C}^{7}$ but that every sphere $\mathbb{S}^{n}$ admits a totally real immersion in $\mathbb{C}^{n}$.

Weinstein $[18$, p. 26] observed that if we take

$$
\mathbb{S}^{n}=\left\{\left(x_{1}, \ldots, x_{n+1}\right) \in \mathbb{R}^{n+1}:|x|^{2}=x_{1}^{2}+\cdots+x_{n+1}^{2}=1\right\},
$$

then the map $\varphi: \mathbb{C}^{n+1} \rightarrow \mathbb{C}^{n}$ given by

$$
\varphi(z)=\left(z_{1}\left(1+2 i z_{n+1}\right), \ldots, z_{n}\left(1+2 i z_{n+1}\right)\right)
$$

restricts to $\mathbb{S}^{n}$ as a Lagrangian immersion of the sphere into $\mathbb{C}^{n}$ that is oneto-one except that the two poles $p^{ \pm}=(0, \ldots, 0, \pm 1)$ are both taken to the origin. That $\varphi$ is a Lagrangian immersion means that if $\vartheta$ is the $(1,1)$-form on $\mathbb{C}^{n}$ given by

$$
\vartheta=\sum_{j=1}^{n} d z_{j} \wedge d \bar{z}_{j},
$$

then $\varphi^{*} \vartheta=0$. It follows that the image, $\Sigma$, of $\mathbb{S}^{n}$ under $\varphi$ is an immersed

2000 Mathematics Subject Classification: Primary 32D10.

Key words and phrases: envelope of holomorphy.

$\left({ }^{1}\right)$ In [14] the methods used are those of differential topology, so it is not evident from that paper whether the envelopes of holomorphy in question may be homeomorphic to domains in $\mathbb{C}^{n}$. By using topological intersection theory as given in [4], it can be shown that, in fact, these domains are not even topologically equivalent to domains in $\mathbb{C}^{n}$. Details are given in Section 3 below. 
totally real submanifold of $\mathbb{C}^{n}$. At the origin of $\mathbb{C}^{n}$, the two local branches of $\Sigma$ meet transversally.

Thus, though among the spheres $\mathbb{S}^{n}$ only the one-sphere and the threesphere embed as totally real submanifolds in $\mathbb{C}^{n}$, each sphere $\mathbb{S}^{n}$ admits a very simple totally real immersion in $\mathbb{C}^{n}$.

2. The main construction. We construct the desired domain as follows.

Fix once and for all an integer $n \geq 2$. Let

$$
\check{\mathbb{S}}^{n}=\left\{z \in \mathbb{C}^{n+1}: z_{1}^{2}+\cdots+z_{n+1}^{2}=1\right\} .
$$

The map $\varphi$ defined in (1) above is of maximal rank on the sphere $\mathbb{S}^{n}$, so there is a neighborhood $U^{*}$, which we fix at the outset, on which $\varphi$ is regular. That is, the pair $\left(U^{*}, \varphi\right)$ is a Riemann domain spread over $\mathbb{C}^{n}$. In what follows, all our constructions are carried out inside $U^{*}$, though we shall not again refer to this restriction.

For positive $r$ and with $|\cdot|$ the Euclidean norm on $\mathbb{C}^{n+1}$, let

$$
\Delta(r)=\left\{z \in \check{\mathbb{S}}^{n}:\left|z-p^{+}\right|<r\right\},
$$

which is a strictly pseudoconvex domain with smooth boundary in $\check{\mathbb{S}}^{n}$, provided $r$ is small enough. Fix $r>0$ small enough that $\varphi$ carries $\overline{\Delta(r)}$ injectively into $\mathbb{C}^{n}$. Having fixed $r$, fix an $r^{\prime} \in(0, r)$.

For a compact set $X$ in $\mathbb{C}^{n}$, we denote by $\mathscr{C}(X)$ the space of continuous $\mathbb{C}$-valued functions on $X$, and by $\mathscr{P}(X)$ the closed subalgebra of $\mathscr{C}(X)$ consisting of those functions that can be approximated uniformly on $X$ by polynomials.

For any $s \geq 0$, the set $\mathbb{S}^{n} \cup \overline{\Delta(s)}$ is polynomially convex and satisfies

$$
\mathscr{P}\left(\mathbb{S}^{n} \cup \overline{\Delta(s)}\right)=\left\{f \in \mathscr{C}\left(\mathbb{S}^{n} \cup \overline{\Delta(s)}\right): f \mid \Delta(s) \text { is holomorphic }\right\},
$$

because it is the union of a compact subset, $\mathbb{S}^{n}$, of $\mathbb{R}^{n+1}$ and a compact, polynomially convex subset, $\overline{\Delta(s)}$, of $\mathbb{C}^{n+1}$ that is invariant under the conjugation $x+i y \mapsto x-i y$ on $\mathbb{C}^{n+1}=\mathbb{R}^{n+1}+i \mathbb{R}^{n+1}$, so that a theorem of Smirnov and Chirka [11] shows the set to be polynomially convex. The polynomial convexity assertion and the equality of the two algebras are given in $[15$, Th. 8.1.26, p. 392]. (In order for this result to apply in the present situation, we need to have the approximation result that

$$
\mathscr{P}(\overline{\Delta(r)})=\{f \in \mathscr{C}(\overline{\Delta(r)}): f \mid \Delta(r) \text { is holomorphic }\} .
$$

This equality is correct: $\Delta(r)$ is a strictly pseudoconvex domain with smooth boundary in the Stein manifold $\check{\mathbb{S}}^{n}$, so if $f$ is continuous on $\overline{\Delta(r)}$ and holomorphic on $\Delta(r)$, then it can be approximated uniformly on $\overline{\Delta(r)}$ by functions $g$ holomorphic on a neighborhood in $\check{\mathbb{S}}^{n}$ of $\overline{\Delta(r)}$. Moreover, the domain $\Delta(r)$ is defined by a strictly plurisubharmonic exhaustion function for $\check{\mathbb{S}}^{n}$, 
so the set $\overline{\Delta(r)}$ is convex with respect to the algebra $\mathscr{O}\left(\check{\mathbb{S}}^{n}\right)$, whence the approximating functions $g$ can be approximated on $\overline{\Delta(r)}$ by functions $h$ holomorphic on the whole of $\check{\mathbb{S}}^{n}$. These functions $h$ can be extended to functions holomorphic on the whole ambient $\mathbb{C}^{n}$ and so can be approximated on the polynomially convex set $\overline{\Delta(r)}$ by polynomials. The desired equality (2) follows.)

Consider now the set $E=\left(\varphi^{-1}\left(\varphi\left(\mathbb{S}^{n}\right)\right) \cap b \Delta(r)\right) \backslash \mathbb{S}^{n}$, a certain compact subset of $b \Delta(r)$.

LeMma 1. If $r$ is small, the set $E$ is polynomially convex.

Proof. When $s$ is small, each branch of the set $\varphi^{-1}\left(\varphi\left(\mathbb{S}^{n}\right)\right) \cap \Delta(s)$ is a totally real smooth manifold that is nearly a disc, whence each compact subset of it is polynomially convex and admits approximation of continuous functions by polynomials. Thus, $E$ is polynomially convex as desired.

Let $U_{0}$ be an open subset of $\check{\mathbb{S}}^{n}$ that contains $E$ and satisfies $\widehat{\bar{U}}_{0}=\bar{U}_{0}$ if $\widehat{\bar{U}}_{0}$ denotes the polynomially convex hull of $\bar{U}_{0}$ and that is so small that $\widehat{\bar{U}}_{0}$ is disjoint from $\overline{\Delta\left(r^{\prime}\right)}$. Let $U_{1}$ be a second neighborhood in $\check{\mathbb{S}}^{n}$ of $E$ with the property that the polynomially convex hull $\widehat{\bar{U}}_{1}$ is contained in $U_{0}$.

LEMMA 2. There is a bounded holomorphic function $g$ on $\Delta(r)$ with $|g|<1$ on $\Delta(r) \backslash \bar{U}_{0}$ and with the nonempty level set $\Sigma_{\alpha}=\{z \in \Delta(r)$ : $|g(z)|=\alpha\}$ contained in $U_{0}$ for certain $\alpha>1$.

Proof. By the embedding theorem of Fornæss and Henkin [5], there exist a strictly convex domain $W$ in $\mathbb{C}^{N}$ for some sufficiently large $N$ and a biholomorphic embedding $\psi$ of a neighborhood of $\bar{\Delta}(r)$ as a complex submanifold $V$ of a neighborhood of $\bar{W}$ such that $V$ is transversal to $b W$ and $\psi^{-1}(W)=\Delta(r)$. Let $U_{1}^{\prime}$ be a bounded open subset of $\mathbb{C}^{N}$ whose intersection with $V$ is $\psi\left(U_{1}\right)$, and let $U_{0}^{\prime}$ be a bounded open subset of $\mathbb{C}^{N}$ whose intersection with $V$ is $\psi\left(U_{0}\right)$. We suppose $\widehat{U_{1}^{\prime}}$ to be contained in $U_{0}^{\prime}$.

Let $\mu$ be a continuous function on $b W$ such that $1 \leq \mu \leq 2$ and $\mu=1$ on $b W \backslash U_{0}^{\prime}$ and $\mu=2$ on $U_{1} \cap b W$ and $1<\mu<2$ on $\left(U_{0} \backslash \bar{U}_{1}\right) \cap b W$. By a theorem of E. Løw [10] there is a function $\widetilde{g}$ bounded and holomorphic on $W$ and vanishing at the point $\psi\left(p^{+}\right)$with the property that the almost everywhere existent boundary values $\widetilde{g}^{*}$ of $\widetilde{g}$ satisfy $\left|\widetilde{g}^{*}\right|=\mu$ almost everywhere with respect to surface area measure on $b W$. For each $\alpha \geq 0$, let $\widetilde{\Sigma}_{\alpha}=\{z \in W$ : $|\widetilde{g}(z)|=\alpha\}$.

To complete the proof of Lemma 2, we need a further lemma:

Lemma 3. If $\alpha \in(1,2)$ is sufficiently close to 2 , then $\widetilde{\Sigma}_{\alpha} \subset U_{0}^{\prime}$.

Proof. Assume the lemma false, i.e., for a sequence $\left\{\alpha_{j}\right\}_{j=1}^{\infty}$ increasing to 2 the set $W \backslash U_{0}^{\prime}$ contains a point $w_{j}$ of $\widetilde{\Sigma}_{\alpha}$. The convexity of $b W$ implies that 
if $1 \leq s<2$ and $C_{s}=\{z \in b W: \mu(z) \leq s\}$, then for $\alpha>s, \overline{\widetilde{\Sigma}}_{\alpha} \cap C_{s}=\emptyset$. This implies that when $j$ is large $\overline{\widetilde{\Sigma}}_{\alpha_{j}} \cap b W \subset \bar{U}_{1}^{\prime}$, which yields $\overline{\widetilde{\Sigma}}_{\alpha_{j}} \subset \widehat{U_{1}^{\prime}}$ when $j$ is large. By hypothesis, $\widehat{U_{1}^{\prime}} \subset U_{0}^{\prime}$. This completes the proof of Lemma 3.

To conclude the proof of Lemma 2, we take $g=\widetilde{g} \circ \psi$. The lemma is proved.

Fix permanently an $\alpha$ as in Lemma 2 .

Notice that the surface $\Sigma_{\alpha}$ is fibered by the analytic hypersurfaces $g^{-1}(\zeta)$ for $\zeta$ with $|\zeta|=\alpha$.

As already noted, $\mathbb{S}^{n} \cup \overline{\Delta(s)}$ is polynomially convex.

If $s \in\left(0, r^{\prime}\right)$, there is a thin solid tube $T$ in $\check{\mathbb{S}}^{n}$ over $\mathbb{S}^{n} \backslash \Delta(s)$ on which $\varphi$ is injective. (The general principle here is that if $h: \mathscr{M} \rightarrow \mathscr{M}^{\prime}$ is a local homeomorphism from the manifold $\mathscr{M}$ to the manifold $\mathscr{M}^{\prime}$ that is injective on the compact set $K \subset \mathscr{M}$, then $h$ is injective on a neighborhood of $K$.) Choose an $r^{\prime \prime} \in\left(r^{\prime}, r\right)$. Let $\Omega_{2}$ be a strictly pseudoconvex domain with

$$
\mathbb{S}^{n} \cup \Delta\left(r^{\prime \prime}\right) \subset \Omega_{2} \Subset(T \cup \Delta(r)) .
$$

The existence of such a domain follows from the polynomial convexity of $\mathbb{S}^{n} \cup \overline{\Delta\left(r^{\prime}\right)}$. We choose $\Omega_{2}$ so large that $b \Omega_{2} \cap\{z \in \Delta(r):|g(z)|>\alpha\}$ is a neighborhood in $b \Omega_{2}$ of $b \Omega_{2} \cap\left(\varphi^{-1}\left(\varphi\left(\mathbb{S}^{n}\right)\right) \backslash \mathbb{S}^{n}\right)$.

The map $\varphi$ carries $\left(b \Omega_{2} \cap \Delta(r)\right) \backslash\{z \in \Delta(r):|g(z)|>\alpha\}$ injectively onto a set $X$ in $\mathbb{C}^{n}$ that is at positive distance from $\varphi\left(S^{n}\right)$.

Let $T^{\prime}$ be a solid tube around $\mathbb{S}^{n}$ in $\check{\mathbb{S}}^{n}$ that is so thin that $T^{\prime} \subset T \cup \Delta\left(r^{\prime}\right)$ and $\varphi\left(T^{\prime}\right)$ is disjoint from the set $X$. In addition, let $r^{\prime \prime \prime}$ be a small positive number slightly greater than $r$. Then let $\Omega_{2}^{\prime}$ be a strictly pseudoconvex domain in $\check{\mathbb{S}}^{n}$ that contains $\mathbb{S}^{n} \cup \overline{\Delta(r)}$ and that is contained in $T^{\prime} \cup \Delta\left(r^{\prime \prime \prime}\right)$. The domain $\Omega_{2}^{\prime}$ can be chosen so that its boundary is transversal to the boundary of $\Omega_{2}$. Using a process detailed in [12], we see that the intersection $b \Omega_{2} \cap b \Omega_{2}^{\prime}$ can be smoothed so as to obtain a strictly pseudoconvex domain $\Omega_{1}^{\prime}$ contained in $\Omega_{2} \cap \Omega_{2}^{\prime}$ and that agrees with this intersection outside a thin neighborhood of $b \Omega_{2} \cap b \Omega_{2}^{\prime}$. The strictly pseudoconvex domain $\Omega_{1}^{\prime}$ is contained in $\check{\mathbb{S}}^{n}$, contains $\mathbb{S}^{n} \cup \Delta\left(r^{\prime}\right)$, and satisfies

$$
\Omega_{1}^{\prime} \cap \Delta(r)=\Omega_{2} \cap \Delta(r) .
$$

Let $\Omega_{1}$ be the domain

$$
\Omega_{1}=\Omega_{1}^{\prime} \backslash\{z \in \Delta(r):|g(z)| \geq \alpha\} .
$$

The domain $\Omega_{1}$ is pseudoconvex and has the following extension property:

Lemma 4. If $V \subset \Omega_{1}$ is a connected open set such that $\bar{V}$ is a neighborhood of the set

$$
\Gamma=b \Omega_{1} \backslash\{z \in \Delta(r):|g(z)|=\alpha\}
$$


in $\Omega_{1} \cup \Gamma$, then each function $f$ holomorphic on $V$ continues holomorphically into $\Omega_{1}$.

Proof. We consider first the set $\widetilde{\Omega}_{1}=\Omega_{1} \backslash \bar{U}_{0}=\Omega_{1}^{\prime} \backslash \bar{U}_{0}$. Its boundary is

$$
b \widetilde{\Omega}_{1}=\left(b \Omega_{1} \backslash \bar{U}_{0}\right) \cup \overline{\Omega_{1}^{\prime} \cap b U_{0}}=S \cup K .
$$

The polynomially convex hull of the compact subset $K$ of the boundary of $\widetilde{\Omega}_{1}$ is contained in the set $\bar{U}_{0}$ and so is disjoint from $\widetilde{\Omega}_{1}$. Accordingly, the principal result of the paper [9] implies that every CR-function on $S$ extends holomorphically through all of $\widetilde{\Omega}_{1}$. If instead of a CR-function on $S$, we are given a function $f$ holomorphic on a one-sided neighborhood of $S$ that lies in $\widetilde{\Omega}_{1}$, then we apply this same extension result to the restriction of $f$ to a surface $S^{\prime}$ lying in $\widetilde{\Omega}_{1}$ and obtained by pulling $S$ in slightly, leaving it fixed at $b S=K \cap b \Omega_{1}$, so that $f$ is defined on $S^{\prime}$.

What we know, then, is that each function holomorphic on the domain $V$ above extends holomorphically into $\widetilde{\Omega}_{1}$. We have to see that there actually is an extension into all of $\Omega_{1}$.

To this end, notice that since the set $\bar{U}_{0}$ is polynomially convex, there is a Stein domain $D$ that consists of $\Omega_{1} \backslash \widetilde{\Omega}_{1}$ together with a thin neighborhood of $b \widetilde{\Omega}_{1} \cap \Omega_{1}$. We can choose the domain $D$ so that $b D \backslash \Sigma_{\alpha}$ is a smooth strictly pseudoconvex surface $S^{\prime}$. A function defined on $\widetilde{\Omega}_{1} \cup V$ is defined on $V \cap D$ and on a neighborhood of $b D \cap \widetilde{\Omega}_{1}$. The function $g$ is defined on a Stein neighborhood of $\bar{D}$, viz. $\Delta(r)$, which is biholomorphically equivalent to a domain in $\mathbb{C}^{n}$.

At this point, it is convenient to treat the case $n \geq 3$ separately from the case $n=2$. Suppose then that $n \geq 3$. Notice that the set $T_{\alpha}=b D \cap$ $\Sigma_{\alpha}$ has the convexity property that if $z \in \bar{D} \backslash T_{\alpha}$, then there are analytic varieties of dimension $n-1$ in a neighborhood of $\bar{D}$ that pass through the point $z$ and miss $T_{\alpha}$, e.g., the level set of $g$ through $z$. This convexity property implies that each CR-function on $b D \backslash T_{\alpha}$ continues holomorphically into $D$, and indeed that any function defined and holomorphic on a one-sided neighborhood of $b D \backslash T_{\alpha}$ in $\bar{D}$ continues through $D$. For this relatively simple result, one can consult [3, Theorem 4.5.2] or [13, Theorem II.3].

The case $n=2$ requires something different. In essence, it seems to be necessary to revisit the ideas used in [9] and by other authors cited there. We begin with the remark that since $\Delta(r)$ is biholomorphically equivalent to a domain in $\mathbb{C}^{2}$, there are global holomorphic coordinates, say $z=\left(z_{1}, z_{2}\right)$, defined on $\Delta$. As the function $g$ is holomorphic on $\Delta(r)$, which is a domain of holomorphy in the $z$-space, there is a factorization

$$
g(z)-g(w)=g_{1}(z, w)\left(z_{1}-w_{1}\right)+g_{2}(z, w)\left(z_{2}, w_{2}\right)
$$

with $g_{1}, g_{2}$ holomorphic but not necessarily bounded on $\Delta(r) \times \Delta(r)$. 
Denote by $K_{\mathrm{BM}}$ the Bochner-Martinelli kernel so that

$$
K_{\mathrm{BM}}(z, w)=c_{2} \frac{\left[\left(\bar{z}_{2}-\bar{w}_{2}\right) d \bar{z}_{1}-\left(\bar{z}_{1}-\bar{w}_{1}\right) d \bar{z}_{2}\right] \wedge \omega(z)}{|z-w|^{4}},
$$

in which $\omega(z)=d z_{1} \wedge d z_{2}$ and $c_{2}$ is a suitable constant. This kernel has the property that if $F$ is holomorphic on the smoothly bounded domain $W$ and is continuous on the closure $\bar{W}$, then for $w \in D$,

$$
F(w)=\int_{b D} f(z) K_{\mathrm{BM}}(z, w) .
$$

Direct calculation shows that

$$
\begin{aligned}
& \bar{\partial}_{z}\left(c_{2} \frac{\bar{z}_{1}-\bar{w}_{1}}{|z-w|^{2}}\right) \omega(z)=\left(z_{2}-w_{2}\right) K_{\mathrm{BM}}(z, w), \\
& \bar{\partial}_{z}\left(c_{2} \frac{\bar{z}_{2}-\bar{w}_{2}}{|z-w|^{2}}\right) \omega(z)=-\left(z_{1}-w_{1}\right) K_{\mathrm{BM}}(z, w) .
\end{aligned}
$$

Consequently, the form

$$
\vartheta(z, w)=c_{2}\left(g_{2}(z, w) \frac{\bar{z}_{1}-\bar{w}_{1}}{|z-w|^{2}}-g_{1}(z, w) \frac{\bar{z}_{2}-\bar{w}_{2}}{|z-w|^{2}}\right) \omega(z)
$$

satisfies

$$
\bar{\partial}_{z} \vartheta(z, w)=(g(z)-g(w)) K_{\mathrm{BM}}(z, w)
$$

and thus, where $g(z) \neq g(w)$, we have

$$
\bar{\partial}_{z}\left\{\frac{\vartheta(z, w)}{g(z)-g(w)}\right\}=K_{\mathrm{BM}}(z, w) .
$$

We now consider the domain $D$ constructed above and a function $f$ defined on a one-sided neighborhood $W$ of $b D \backslash T_{\alpha}$. Our goal is to show that $f$ continues holomorphically into the whole of $D$. We shall assume that, in fact, $f$ is defined and holomorphic on a neighborhood of $b D \backslash T_{\alpha}$. This is a matter of convenience: If $f$ is not defined on such a neighborhood, replace $D$ by a domain $D^{\prime}$ obtained by pulling $b D \backslash T_{\alpha}$ in a little, leaving $T_{\alpha}$ fixed. The original $f$ is now defined on a neighborhood of $b D^{\prime} \backslash T_{\alpha}$, and we need only show that $f$ continues into $D^{\prime}$.

Accordingly, define a function $H$ on $D$ as follows. For $w \in D$, let $|g(w)|=\beta$. We have $\beta<\alpha$. Choose $\gamma \in(\beta, \alpha)$ such that the level set $\Sigma_{\gamma}=\{z \in \Delta(r):|g(z)|=\gamma\}$ is a smooth hypersurface that meets $b D \backslash T_{\alpha}$ transversally. By Stokes's theorem, the quantity

$$
H_{\gamma}(w)=\int_{b D \cap\{z:|g(z)|<\gamma\}} f(z) K_{\mathrm{BM}}(z, w)+\int_{b D \cap \Sigma_{\gamma}} \frac{f(z) \vartheta(z, w)}{g(z)-g(w)}
$$

is independent of the choice of $\gamma$. (In the expression for $H_{\gamma}(w)$, the orientation of $b D \cap\{z: \mid g(z)<\gamma\}$ is that induced on $b D$ as the boundary of 
the domain $D$. The orientation on $b D \cap \Sigma_{\gamma}$ is taken to be that induced on $b D \cap \Sigma_{\gamma}$ as the boundary of the manifold $\Sigma_{\gamma} \cap D$. The latter manifold is taken to be oriented as part of the boundary of $D \cap\{z:|g(z)|<\gamma\}$.) We define $H(w)$ to be $H_{\gamma}(w)$. The function $H$ defined in this way depends in a real-analytic way on the point $w$ in $D$.

Denote by $m$ the minimum value of $|g|$ on $\bar{D}$. The set $M$ on which $|g|$ assumes the value $m$ is a compact subset of $b D \backslash T_{\alpha}$, and consequently, if $\varepsilon>0$ is sufficiently small, $f$ is defined and holomorphic on the set $B=$ $\{z \in \bar{D}:|g(z)| \leq m+\varepsilon\}$. If $\varepsilon$ is chosen properly-invoke Sard's theoremthen the level set $\Sigma_{m+\varepsilon}$ will be transversal to $b D$, and we can use Stokes's theorem to write that, for $w \in B \cap D$,

$$
H(w)=H_{m+\varepsilon}(w)=\int_{b B} f(z) K_{\mathrm{BM}}(z, w)=f(w) .
$$

That is to say, we have a real-analytic function $H$ on $D$ that agrees with $f$ on an open set in $D$. It follows that $H$ is holomorphic on $D$ and that it gives the holomorphic continuation of $f$ through $D$.

We have now a complete proof of Lemma 4.

Lemma 5. The map $\varphi$ is injective on the set $\Gamma$ defined in the preceding lemma.

Proof. The map $\varphi$ is injective on $\Delta(r)$ and on $T^{\prime}$, so if $\varphi(z)=\varphi\left(z^{\prime}\right)$ for $z, z^{\prime} \in \Gamma$, then $z \in \Delta(r)$ and $z^{\prime} \in T^{\prime} \backslash \Delta(r)$ or vice versa. Suppose the former case to obtain. As $z \in \Delta(r)$, we have $\varphi(z) \in X$. Finally, $z^{\prime} \in T^{\prime}$ implies that $\varphi\left(z^{\prime}\right) \notin X$. This completes the proof.

The fact that $\varphi$ is injective on $\Gamma$ implies that if $\Omega_{0}$ is a thin one-sided neighborhood of $\Gamma$ contained in $\Omega_{1}$, then $\Omega_{0}$ is carried injectively by $\varphi$ onto a domain $\Omega$ in $\mathbb{C}^{n}$. As each $f$ holomorphic on $\Omega_{0}$ extends holomorphically into the pseudoconvex domain $\Omega_{1}$, the envelope of holomorphy of $\Omega$ is the Riemann domain $\left(\Omega_{1}, \varphi\right)$. The manifold $\Omega_{1}$ contains the totally real sphere $\mathbb{S}^{n}$.

Thus, for every $n=2,3, \ldots$, we have found a domain, say $\mathcal{D}_{n}$, in $\mathbb{C}^{n}$ whose envelope of holomorphy, $\widehat{\mathcal{D}}_{n}$, is a neighborhood of the $n$-sphere $\mathbb{S}^{n}$ in the complexified $n$-sphere $\check{\mathbb{S}}^{n}$.

There are various cases:

(1) $n=3$. It was noted by Gromov that the three-sphere $\mathbb{S}^{3}$ admits totally real embeddings in $\mathbb{C}^{n}$; explicit embeddings were constructed by Ahern and Rudin [2]. Such an embedding, if chosen to be real-analytic, extends to a biholomorphic embedding of a neighborhood of $\mathbb{S}^{3}$ in $\check{\mathbb{S}}^{3}$ into $\mathbb{C}^{3}$, so if the domain $\mathcal{D}_{3}$ is chosen to be sufficiently thin, the envelope $\widehat{\mathcal{D}}_{3}$ is biholomorphically equivalent to a domain in $\mathbb{C}^{3}$. 
(2) $n=7$. Again, it was noted by Gromov that the seven-sphere $\mathbb{S}^{7}$ does not admit a totally real embedding into $\mathbb{C}^{7}$. Details of an argument establishing this are given in [17]. It follows that the envelope $\widehat{\mathcal{D}}_{7}$ is not biholomorphically equivalent to a domain in $\mathbb{C}^{7}$. It is, however, real-analytically equivalent to such a domain, for the complexification $\breve{S}^{7}$ is bianalytically equivalent to the product $\mathbb{S}^{7} \times \mathbb{R}^{7}$, which, in turn, is bianalytically equivalent to $\left(\mathbb{R}^{8} \backslash\{0\}\right) \times \mathbb{R}^{6}$. See $[17]$.

(3) $n \neq 1,3,7$. For such $n$, the sphere $\mathbb{S}^{n}$ does not embed as a totally real submanifold of $\mathbb{C}^{n}$. The case of even $n$ was treated by Wells [19] and by Aeppli [1]; the general case is in [17]. It follows that for $n \neq 1,3,7$, the envelope $\widehat{\mathcal{D}}_{n}$ is not biholomorphically equivalent to a domain in $\mathbb{C}^{n}$. In the case of the even-dimensional spheres more is true: If $n$ is even, then results of Aeppli [1] imply that no Stein tube over $\mathbb{S}^{n}$ embeds homeomorphically in $\mathbb{C}^{n}$, so from this, when $n$ is even, the envelope $\widehat{\mathcal{D}}_{n}$ is not homeomorphic to a domain in $\mathbb{C}^{n}$. The case of odd-dimensional spheres is not covered in the paper [1].

It is true, though, that for odd $n$, the envelope $\widehat{\mathcal{D}}_{n}$ is not diffeomorphic to a domain in $\mathbb{C}^{n}$. This is an immediate consequence of the known resultsee Kervaire [8] and the references cited there - that the normal bundle of a smoothly embedded $n$-sphere in $\mathbb{R}^{2 n}$ is trivial. Suppose then that $\widehat{\mathcal{D}}_{n}$ is diffeomorphic to a domain in $\mathbb{C}^{n}$ under, say, the diffeomorphism $\psi$. Then the normal bundle to the embedded sphere $\psi\left(\mathbb{S}^{n}\right)$ in $\mathbb{C}^{n}$ is trivial, which implies that the normal bundle of the embedded sphere $\mathbb{S}^{n}$ in $\widehat{\mathcal{D}}_{n}$ (or $\breve{\mathbb{S}}^{n}$ ) is trivial. The complex structure $J$ on $\check{\mathbb{S}}^{n}$ effects an isomorphism of the normal bundle to $\mathbb{S}^{n}$ with the tangent bundle to $\mathbb{S}^{n}$. Consequently, $\mathbb{S}^{n}$ is parallelizable, so $n=1,3$, or 7 . (This argument was already used in [17].)

This discussion is again in the domain of differential topology; whether $\mathcal{D}_{n}, n$ odd, not $1,3,7$, is homeomorphic to a domain in $\mathbb{C}^{n}$ is still not evident.

3. The envelope of holomorphy constructed in [14] is not homeomorphic to a domain in $\mathbb{C}^{n}$. In the paper [14] a domain $\Omega$ in $\mathbb{C}^{n}, n \geq 2$, is exhibited whose envelope of holomorphy $\widetilde{\Omega}$ is not diffeomorphic - even of class $\mathscr{C}^{1}$ - to a domain in $\mathbb{C}^{n}$. At the time that paper was written, it was not evident to the author that the Riemann domain $\widetilde{\Omega}$ is not homeomorphic to a domain in $\mathbb{C}^{n}$. The object of the present paragraph is to observe that, in fact, $\widetilde{\Omega}$ is not topologically equivalent to a domain in $\mathbb{C}^{n}$.

We begin by recalling the principle involved in the example given in [14]. There the counterexample hinges on the construction of a domain $\Omega$ in $\mathbb{C}^{n}$ such that if $(\widetilde{\Omega}, \pi)$ is the envelope of holomorphy of $\Omega$, then the Riemann domain $\widetilde{\Omega}$ contains a pair of smoothly embedded orientable $n$-manifolds $M_{1}$ 
and $M_{2}$ that intersect in a single point and whose intersection is transversal. By intersection theory in the setting of differential topology (see [7, p. 132]) this configuration cannot exist in $\mathbb{C}^{n}$. This is an argument in differential topology and does not exclude the possibility that $\widetilde{\Omega}$ might be homeomorphic to a domain in $\mathbb{C}^{n}$.

There is a topological theory of intersection that can be brought to bear on the matter at hand and that yields the result we seek: The manifold $\widetilde{\Omega}$ is not homeomorphic to a domain in $\mathbb{C}^{n}$. The intersection theory necessary for this conclusion is written out in the book of Dold [4, pp. 197-201 and $342-345]$.

In our situation, this theory attaches to each pair $\xi \in H_{i}\left(M_{1}\right)$ and $\eta \in H_{j}\left(M_{2}\right)$ of homology classes a homology class $\xi \bullet \eta \in H_{i+j-2 n}\left(M_{1} \cap M_{2}\right)$. With $i=j=n$ and with $\xi$ and $\eta$ the fundamental classes $o_{M_{1}} \in H_{n}\left(M_{1}\right)$ and $o_{M_{2}} \in H_{n}\left(M_{2}\right)$, the resulting product $o_{M_{1}} \bullet o_{M_{2}}$ lies in $H_{0}\left(M_{1} \cap M_{2}\right)=$ $H_{0}(\{p\})=\mathbb{Z}$. Moreover, because the manifolds $M_{1}$ and $M_{2}$ meet transversally at the point $p$, we have $o_{M_{1}} \bullet o_{M_{2}}= \pm o_{\{p\}}$. In particular, this product is not zero.

On the other hand, these intersection numbers are altered at most by a sign by a homeomorphism of the manifold $\widetilde{\Omega}$, so because in $\mathbb{R}^{n}$ all intersection products vanish (see [4, p. 198]), the manifold $\widetilde{\Omega}$ cannot be homeomorphic to a domain in $\mathbb{C}^{n}$.

4. Another example. To conclude, we give an example that was brought to our attention by William R. Zame. The paper [16] contains an example of a domain $D$ in $\mathbb{C}^{n}$ whose universal covering space $D^{*}$ is not biholomorphic to a domain in $\mathbb{C}^{n}$. The obstruction is that by construction $D^{*}$ contains a pair of smoothly embedded $n$-manifolds $\Sigma$ and $\Sigma_{1}$ that intersect transversally at one point and that have no other intersection. The existence of these manifolds precludes the possibility that $D^{*}$ is biholomorphic or even diffeomorphic to a domain in $\mathbb{C}^{n}$. And, as in the preceding section, we recognize that $D^{*}$ is not topologically a domain in $\mathbb{C}^{n}$. If we now recall that according to $[6]$, there is a domain $D_{0}$ in $\mathbb{C}^{n}$ whose envelope of holomorphy is the manifold $D^{*}$, we have another example of a domain in $\mathbb{C}^{n}$ whose envelope of holomorphy is not homeomorphic to a domain in $\mathbb{C}^{n}$.

\section{References}

[1] A. Aeppli, On determining sets in a Stein manifold, in: Proc. Conf. Complex Analysis (Minneapolis 1964), Springer, Berlin, 1965, 48-58.

[2] P. Ahern and W. Rudin, Totally real embeddings of $S^{3}$ in $\mathbb{C}^{3}$, Proc. Amer. Math. Soc. 94 (1985), 460-462. 
[3] E. M. Chirka and E. L. Stout, Removable singularities in the boundary, in: Contributions to Complex Analysis and Analytic Geometry, Aspects of Math. E26, Vieweg, Braunschweig, 1994, 43-104.

[4] A. Dold, Lectures on Algebraic Topology, Grundlehren Math. Wiss. 200, Springer, New York, 1972.

[5] J. E. Fornæss, Embedding strictly pseudoconvex domains in convex domains, Amer. J. Math. 98 (1976), 529-569.

[6] J. E. Fornæss and W. R. Zame, Riemann domains and envelopes of holomorphy, Duke Math. J. 50 (1983), 273-283.

[7] M. W. Hirsch, Differential Topology, Grad. Texts in Math. 33, Springer, New York, 1976.

[8] M. A. Kervaire, Sur le fibré normal à une variété plongée dans l'espace euclidien, Bull. Soc. Math. France 87 (1959), 397-401.

[9] C. Laurent-Thiébaut, Sur l'extension des fonctions CR dans une variété de Stein, Ann. Mat. Pura Appl. (4) 150 (1988), 141-151.

[10] E. Løw, Inner functions and boundary values in $H^{\infty}(\Omega)$ and $A(\Omega)$ in smoothly bounded pseudoconvex domains, Math. Z. 185 (1984), 191-210.

[11] M. M. Smirnov and E. M. Chirka, Polynomial convexity of some sets in $\mathbb{C}^{n}$, Mat. Zametki 50 (1991), no. 5, 81-89 (in Russian); English transl.: Math. Notes 50 (1991), 1151-1157.

[12] E. L. Stout, Interpolation manifolds, in: Recent Developments in Several Complex Variables (Princeton, NJ, 1979), Ann. of Math. Stud. 100, Princeton Univ. Press, Princeton, 1981, 373-391.

[13] - Removable singularities for the boundary values of holomorphic functions, in: Several Complex Variables (Stockholm, 1987/1988), Math. Notes 38, Princeton Univ. Press, Princeton, NJ, 1993, 600-629.

[14] - A domain whose envelope of holomorphy is not a domain, Ann. Polon. Math. 89 (2006), 197-201.

[15] —, Polynomial Convexity, Progr. Math. 261, Birkhäuser Boston, Boston, MA, 2007.

[16] E. L. Stout and W. R. Zame, Totally real imbeddings and the universal convering spaces of domains of holomorphy: Some examples, Manuscripta Math. 50 (1985), $29-48$.

[17] —, - A Stein manifold topologically but not holomorphically equivalent to a domain in $\mathbb{C}^{n}$, Adv. in Math. 60 (1986), 154-160.

[18] A. Weinstein, Lectures on Symplectic Manifolds, CBMS Reg. Conf. Ser. Math. 29, Amer. Math. Soc., Providence, RI, 1977.

[19] R. O. Wells, Jr., Compact real submanifolds of a complex manifold with nondegenerate holomorphic tangent bundles, Math. Ann. 179 (1969), 123-129.

Department of Mathematics

University of Washington

Seattle, WA 98195, U.S.A.

E-mail: stout@math.washington.edu

Received 1.7.2008

and in final form 17.9.2008 